\title{
A Lagrangian Model to Predict Microscallop Motion in non Newtonian Fluids
}

\author{
Yashaswini Murthy ${ }^{1}$ and Ravi Banavar ${ }^{2}$
}

\begin{abstract}
The need to develop models to predict the motion of microrobots, or robots of a much smaller scale, moving in fluids in a low Reynolds number regime, and in particular, in non Newtonian fluids, cannot be understated. The article develops a Lagrangian based model for one such mechanism - a two-link mechanism termed a microscallop, moving in a low Reynolds number environment in a non Newtonian fluid. The modelling proceeds through the conventional Lagrangian construction for a two-link mechanism and then goes on to model the external fluid forces using empirically based models for viscosity to complete the dynamic model. The derived model is then simulated for different initial conditions and key parameters of the non Newtonian fluid, and the results are corroborated with a few existing experimental results on a similar mechanism under identical conditions. Lastly, with a view to implementing control algorithms we explore accessibility of the system at certain configurations.
\end{abstract}

\section{INTRODUCTION}

The ability to access small spaces inside the human body at low Reynolds number (LRN) has facilitated research into controllable micro and nanorobotics. Alongside its myriad applications [1], [2], [3], of particular interest to this work is drug delivery, which in itself is a topic with vast room for technological improvements [4]. To aid in drug delivery and many other minimally invasive applications of micro robots it is important to understand the environment in which they operate. Since most of the fluids in the human body are of the non Newtonian kind, it is necessary to study the properties of such fluids in the LRN regime in order to model microrobots for the same. To this end, the next section discusses certain existing mathematical models which describe the locomotive behaviour of organisms in the regime of interest.

\section{MATHEMATICAL MODELLING}

The study of locomotion of micro organisms in LRN can be broadly classified into two categories: Newtonian and non Newtonian. In order to be able to design and control these microrobots, it is necessary to mathematically model them and understand their dynamical behaviour subject to manipulation of certain parameters. The simplest microrobot capable of locomotion has two rigid links and a single degree of freedom. [5] is an early paper which presents a fundamental theorem, concerning the motion characteristics of a scallop-like structure in the LRN regime. Henceforth, all our discussions are focused on locomotion in LRN regime.

\footnotetext{
${ }^{1}$ Department of Mechanical Engineering, Indian Institute of Technology Bombay, India.

${ }^{2}$ Department of Systems and Control Engineering, Indian Institute of Technology Bombay, India.
}

\section{A. Scallop Theorem}

A body in the LRN regime experiences a greater magnitude of viscous forces as when compared to the inertial forces. Consequentially, motion at that instant is entirely determined by the forces exerted at that moment and by nothing in the past [5].

The Navier Stokes equation, after neglecting the inertia terms, represents such fluid behaviour, and is given by:

$$
\eta \nabla^{2} \mathbf{v}-\nabla p=0 \quad \nabla \cdot \mathbf{v}=0
$$

The above equation is linear in space, and time independent [6], [7]. When applied to LRN locomotion, the linearity and time-independence of the Stokes equation of motion lead to two important properties.

- The first is rate independence: if a body undergoes surface deformation, the distance travelled by the swimmer between two different surface configurations does not depend on the rate at which the surface deformation occurs but only on its geometry (i.e. the sequence of shapes the swimmer passes through between these two configurations) [8].

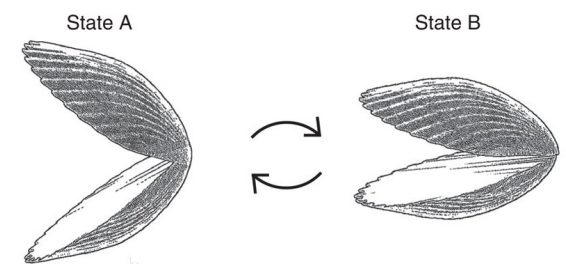

Fig. 1: The open and close configurations of a scallop [9]

- The second important property is the so-called scallop theorem: if the sequence of shapes displayed by a swimmer deforming in a time periodic fashion is identical when viewed after a time-reversal transformation, then the swimmer experiences no motion on an average. The only constraint is on the sequence of configurations of the swimmer and not the rate at which it executes its motion. This class of surface deformations is termed 'reciprocal deformation'. The scallop theorem introduces a strong geometrical constraint on the type of swimming motion which is effective at low Reynolds numbers [8].

Various theories are used to model fluid bodies in the LRN regime. Two amongst these, that are the most commonly employed are the slender body theory [10] and the resistive force theory [11]. These will be discussed in the following section. 


\section{FLUID DYNAMICS AND RESISTIVE FORCE THEORY}

Although non Newtonian fluids can be categorized into various categories based on their shear stress-strain rate behaviour, this paper primarily studies the time independent shear thinning non Newtonian fluids.

A shear thinning fluid is characterized by an apparent viscosity $\eta$ (defined as $\sigma_{x y} / \dot{\gamma}_{x y}$, where $\sigma_{x y}$ is the shear stress and $\dot{\gamma}_{x y}$ is the shear strain rate the fluid is subjected to) which gradually decreases with increasing shear rate. In polymeric systems (melts and solutions), at low shear rates, the apparent viscosity approaches a Newtonian plateau, where the viscosity is independent of shear rate (zero shear viscosity, $\eta_{0}$ ), given by:

$$
\lim _{\dot{\gamma}_{x y} \rightarrow 0} \frac{\sigma_{x y}}{\dot{\gamma}_{x y}}=\eta_{0}
$$

Furthermore, strictly polymer solutions also exhibit a similar plateau at very high shear rates (infinite shear viscosity, $\eta_{\infty}$ ), i.e.,

$$
\lim _{\dot{\gamma}_{x y} \rightarrow \infty} \frac{\sigma_{x y}}{\dot{\gamma}_{x y}}=\eta_{\infty}
$$

There are various empirically developed models to capture the behaviour of shear thinning fluids, a few of which are discussed below:

- Power law model states that the relationship between shear stress $(\sigma)$ and shear rate $(\dot{\gamma})$ plotted on log-log coordinates for a shear-thinning fluid can be approximated by a straight line over an interval of shear rate as,

$$
\sigma=m(\dot{\gamma})^{n}
$$

where, $n$ is the power index and $m$ is a flow consistency index, both of which are characteristics of the fluid.

- Carreau viscosity model is another model which has been found to be more accurate with respect to the experimental results. The viscosity relationship according to such a model is presented below.

$$
\mu_{e f f}(\dot{\gamma})=\mu_{i n f}+\left(\mu_{0}-\mu_{i n f}\right)\left(1+(\lambda \dot{\gamma})^{2}\right)^{\frac{n-1}{2}}
$$

where,

$\mu_{0}$ is viscosity at shear rate tending to zero

$\mu_{\text {inf }}$ is viscosity at shear rate tending to infinity

$\lambda$ is the relaxation time

In the above two laws, it is necessary that $0<n<1$, to have a shear thinning behaviour. At low shear rate, a Carreau fluid behaves as a Newtonian fluid, while at higher shear rate as a power law fluid [12].

Having understood the distinguishing properties of non Newtonian fluids, there are certain aspects of these fluids that can be exploited to overcome the scallop theorem and have non zero displacement with reciprocal motion. Since coefficient of viscosity in a $\mathrm{NN}$ fluid is no longer a constant (it depends on the shear strain rate), the reciprocal theorem is no longer rate independent. The viscous resistive forces acting on the acting on the body will depend on its velocity, in both the backward and forward strokes. Different stroke velocities, lead to different forces in the two directions, yielding an overall nonzero displacement post every periodic set of motions. This rate dependency can be exploited to design a periodic swimmer in a NN fluid. [7]

However, the scallop theorem holds only for a single scallop in a uniform flow field. Two scallops engaging in reciprocal motion, with non trivial phase differences, lead to a non uniform flow field between the two, causing the collapse of the scallop theorem. Hence there is no many-scallop theorem [7].

In order to determine the displacement that these bodies undergo, it is necessary to determine the forces acting on them due to the fluid. To model the forces acting on bodies with high aspect ratio (similar to the most commonly prevalent biological specimens) several theories have been proposed, of which resistive force theory is the most commonly employed.

\section{A. Resistive Force Theory}

Resistive force theory is incorporated to determine viscous resistive forces on bodies whose radius of curvature is of a lower order of magnitude than its length. It provides us with the linear force density as a linear function of the local surface velocity of the filament. This force density is determined in the transverse and longitudinal direction of the filaments. Slender body theory is included at times, to obtain more accurate determination of these resistive forces. To extend this theory to non Newtonian fluids, it is necessary to be able to estimate drag coefficients in such a fluid.

[11] proposes two approaches to calculate the non Newtonian drag coefficients. The first method involves deducing an empirical relation from experiments of sedimenting rods in shear-thinning fluids. The second method involves incorporating the Carreau visocity model to determine the drag coefficients. In order to determine these drag coefficients, it is necessary to determine the local shear strain rate at the surface of the filament, which is the same of the surface velocity (assuming no slip condition). We can then incorporate the shear-thinning nature of the fluid through a correction to the Newtonian drag coefficients and obtain a nonlinear velocity-force relationship. Much of our work on non-Newtonian fluid parameters and resistive force models is incorporated from the papers by Lauga [7], [8] and [11]. Consider a filament of length $2 l$ and radius $a$ as shown in Fig 2

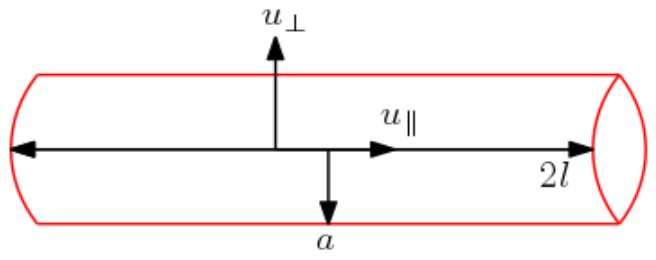

Fig. 2: Straight filament of length $2 l$ and cross-sectional radius $a$ may translate in a fluid along its length (velocity $u_{\|}$) or perpendicular to it $\left(u_{\perp}\right)$ [11] 
The parallel and perpendicular drag coefficients in such a case assuming a locally Newtonian flow is given by [11]:

$$
\begin{gathered}
\left.\frac{f_{\|}}{u_{\|}}\right|_{r=a} \equiv b_{\|}=\frac{4 \pi \mu_{0}}{\ln \left(\frac{4 l^{2}}{a^{2}}\right)-1} \\
\left.\frac{f_{\perp}}{u_{\perp}}\right|_{r=a} \equiv b_{\perp}=\frac{8 \pi \mu_{0}}{\ln \left(\frac{4 l^{2}}{a^{2}}\right)+1}
\end{gathered}
$$

where, $f_{\|}$and $f_{\perp}$ are the linear force densities in the parallel and perpendicular directions respectively. Upon further analysis it can be seen that the average shear rate due to both parallel and perpendicular motion is given by:

$$
\dot{\gamma}_{a v g}=\frac{\sqrt{f_{\perp}^{2}+2 f_{\|}^{2}}}{2 \sqrt{2} a \pi \mu_{0}}
$$

where $\mu_{0}$ is the Newtonian viscosity of the medium [11].

In case of non Newtonian fluids, $\mu_{0}$ is no longer a constant and is a function of strain rate. As a result, to propose drag coefficients to use with the Carreau model (or any emperical non Newtonian fluid model) we require the knowledge of the strain rates in the fluid near the filament.

Consider the Carreau fluid model, whose viscosity relationship is given by:

$$
\mu=\mu_{i n f}+\left(\mu_{0}-\mu_{i n f}\right)\left(1+(\lambda \dot{\gamma})^{2}\right)^{\frac{n-1}{2}}
$$

Since high shear rates are unlikely to occur in our context, we set $\mu_{\text {inf }}=0$, so the model simplifies to

$$
\frac{\mu}{\mu_{0}}=\left(1+(\lambda \dot{\gamma})^{2}\right)^{\frac{n-1}{2}}
$$

With the above simplification, we obtain the non Newtonian drag coefficients to be:

$$
b_{C_{\perp}}=R_{C}\left(u_{\perp}, u_{\|}\right) b_{\perp}, \quad b_{C_{\|}}=R_{C}\left(u_{\perp}, u_{\|}\right) b_{\|}
$$

where $b_{\|}$and $b_{\perp}$ are given by (6) and (7) respectively and $R_{C}\left(u_{\perp}, u_{\|}\right)$is the non Newtonian correction factor defined as below:

$$
R_{C}=\left[1+\left(\frac{\lambda b_{\perp} u_{\dot{\gamma}}}{2 \sqrt{2} a \pi \mu_{0}}\right)^{2}\right]^{\frac{n-1}{2}}
$$

where $u_{\dot{\gamma}}$ is the shear rate velocity defined as:

$$
u_{\dot{\gamma}}=\sqrt{u_{\perp}^{2}+2 \frac{b_{\|}^{2}}{b_{\perp}^{2}} u_{\|}^{2}}
$$

where $u_{\perp}$ and $u_{\|}$are the velocities of the fluid perpendicular and parallel to the slender filament [11]. With this preliminary introduction to fluid force modelling, we move onto the Lagrangian modelling of the scallop.

\section{LAGRAngian Model of the SCALlop}

This section discusses the Lagrange model of the scallop in detail. Fig 3 presents the model of a scallop as two rigid links with a single hinge.

\section{A. System Model}

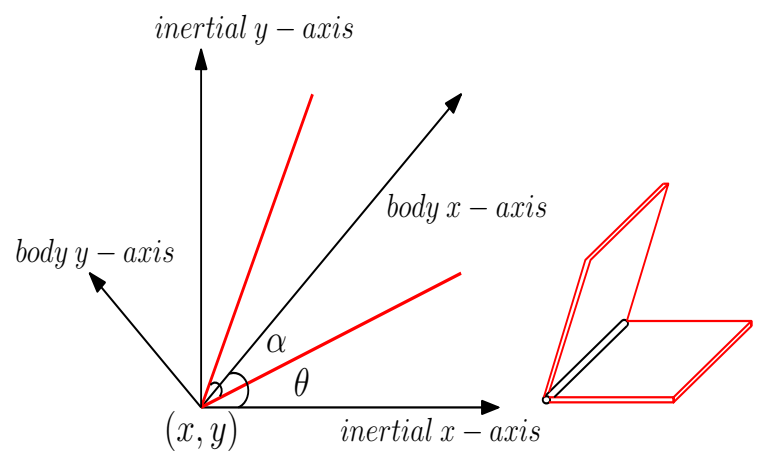

(a)

(b)

Fig. 3: (a)The Schematic of a Scallop. (b)3 Dimensional model of a Scallop

The mass of the square links are $m_{1}$ and $m_{2}$, their lengths (as well as widths) are $L_{1}$ and $L_{2}$ and thicknesses are $a_{1}$ and $a_{2}$. The model has four generalised coordinates, $(x, y, \theta, \alpha)$, $(x, y)$ representing the location of the hinge of the scallop with respect to a fixed coordinate axes, $\theta$, the angle between the scallop body axis and the inertial $x$ axis, and $\alpha$ being the angle between the scallop links. Since there are no conservative forces acting on the scallop, the Lagrangian will consist of only the kinetic energy due to the motion of the links. The force on the links of the scallop are calculated using resistive force theory as shown below.

\section{B. Forces on the links}

The force density, that is the force per unit area, acting on the links is taken as the drag coefficient times the strain rate (the velocity of the link in this case) and wave number [8], [13]. The value of this coefficient varies according to the strain rate, since it is a non Newtonian fluid and is a medium property. The scallop can be assumed to be a rigid segment of a planar sheet whose wavelength is considerably larger than the scallop dimensions. The wave number accounts for the in-extensible planar sheet like structure of the scallop link, keeping the high aspect ratio of the link thickness and length intact. This serves as an extension to the resistive force theory employed in this context. The longitudinal drag coefficient is indicated by $b_{C_{\|}}$and the lateral drag coefficient is by $b_{C_{\perp}}$ and are given by 10 .

The force density on link 1 is given by:

$$
F_{\|}(s)=b_{C_{\|}}(s) u_{\|}(s) k \quad F_{\perp}(s)=b_{C_{\perp}}(s) u_{\perp}(s) k,
$$

where $u_{\|}$and $u_{\perp}$ represent the velocity parallel and perpendicular to the links.

Since $b_{C_{\|}}, b_{C_{\perp}}, v_{\|}, v_{\perp}$ all vary with distance along the length of the link, the forces also vary as a function of position along the length of the link.

The force densities acting on link 1 are found to be,

$$
\begin{aligned}
& F_{x 1}(s)=F_{\|}(s) \cos \left(\theta+\frac{\alpha}{2}\right)-F_{\perp}(s) \sin \left(\theta+\frac{\alpha}{2}\right) \\
& F_{y 1}(s)=F_{\|}(s) \sin \left(\theta+\frac{\alpha}{2}\right)+F_{\perp}(s) \cos \left(\theta+\frac{\alpha}{2}\right)
\end{aligned}
$$


Similarly the force density can be computed for the second link, with the only change being the angle made by the second link with the horizontal axis of the spatial frame. The total force $f_{x 1}$ in the $x$ direction and $f_{y 1}$ in the $y$ direction on link 1 are given by integrating the force density along the length and width of the link, as shown:

$$
f_{x 1}=L_{1} * \int_{0}^{L_{1}} F_{x 1}(s) d s, \quad f_{y 1}=L_{1} * \int_{0}^{L_{1}} F_{y 1}(s) d s
$$

Since we are assuming the force density to vary along the length and be independent of the width, the integration is with respect to the position along the length only. Similarly we can obtain the forces $f_{x 2}$ and $f_{y 2}$ acting on the second link.

With the above computation, the Euler Lagrange Equations corresponding to the four generalised coordinates are derived.

\section{Euler Lagrange Equations}

1) Equation of Motion corresponding to $x$ :

$$
\begin{aligned}
f_{x 1}+ & f_{x 2}=\ddot{x}\left(m_{1}+m_{2}\right)-\frac{\dot{\theta}}{2}\left[m_{1} L_{1} \cos \left(\theta+\frac{\alpha}{2}\right)\left(\dot{\theta}+\frac{\dot{\alpha}}{2}\right)\right. \\
& \left.+m_{2} L_{2} \cos \left(\theta-\frac{\alpha}{2}\right)\left(\dot{\theta}-\frac{\dot{\alpha}}{2}\right)\right]-\frac{\ddot{\theta}}{2}\left[m_{1} L_{1} \sin \left(\theta+\frac{\alpha}{2}\right)\right. \\
& \left.+m_{2} L_{2} \sin \left(\theta-\frac{\alpha}{2}\right)\right]-\frac{\dot{\alpha}}{4}\left[m_{1} L_{1} \cos \left(\theta+\frac{\alpha}{2}\right)\left(\dot{\theta}+\frac{\dot{\alpha}}{2}\right)\right. \\
& \left.-m_{2} L_{2} \cos \left(\theta-\frac{\alpha}{2}\right)\left(\dot{\theta}-\frac{\dot{\alpha}}{2}\right)\right]-\frac{\ddot{\alpha}}{4}\left[m_{1} L_{1} \sin \left(\theta+\frac{\alpha}{2}\right)\right. \\
& \left.-m_{2} L_{2} \sin \left(\theta-\frac{\alpha}{2}\right)\right]
\end{aligned}
$$

where $f_{x 1}$ and $f_{x 2}$ are as described in the previous subsection.

2) Equation of Motion corresponding to $y$ :

$$
\begin{aligned}
f_{y 1}+ & f_{y 2}=\ddot{y}\left(m_{1}+m_{2}\right)-\frac{\dot{\theta}}{2}\left[m_{1} L_{1} \sin \left(\theta+\frac{\alpha}{2}\right)\left(\dot{\theta}+\frac{\dot{\alpha}}{2}\right)\right. \\
& \left.+m_{2} L_{2} \sin \left(\theta-\frac{\alpha}{2}\right)\left(\dot{\theta}-\frac{\dot{\alpha}}{2}\right)\right]+\frac{\ddot{\theta}}{2}\left[m_{1} L_{1} \cos \left(\theta+\frac{\alpha}{2}\right)\right. \\
& \left.+m_{2} L_{2} \cos \left(\theta-\frac{\alpha}{2}\right)\right]-\frac{\dot{\alpha}}{4}\left[m_{1} L_{1} \sin \left(\theta+\frac{\alpha}{2}\right)\left(\dot{\theta}+\frac{\dot{\alpha}}{2}\right)\right. \\
& \left.-m_{2} L_{2} \sin \left(\theta-\frac{\alpha}{2}\right)\left(\dot{\theta}-\frac{\dot{\alpha}}{2}\right)\right]+\frac{\ddot{\alpha}}{4}\left[m_{1} L_{1} \cos \left(\theta+\frac{\alpha}{2}\right)\right. \\
& \left.-m_{2} L_{2} \cos \left(\theta-\frac{\alpha}{2}\right)\right]
\end{aligned}
$$
tion.

where $f_{y 1}$ and $f_{y 2}$ are as described in the previous subsec-

3) Equation of Motion corresponding to $\theta$ : The total torque acting on link 1 is given by:

$$
\tau_{1}=\int_{0}^{L_{1}} s_{1} * F_{\perp}(s) * L_{1} * d s_{1}
$$

A similar expression could be obtained for $\tau_{2}$ acting on the second link.

$$
\begin{aligned}
\tau_{1}+ & \tau_{2}=+\frac{\dot{\theta} \dot{y}}{2}\left[m_{1} L_{1} \sin \left(\theta+\frac{\alpha}{2}\right)+m_{2} L_{2} \sin \left(\theta-\frac{\alpha}{2}\right)\right] \\
+ & \frac{\dot{\theta} \dot{x}}{2}\left[m_{1} L_{1} \cos \left(\theta+\frac{\alpha}{2}\right)+m_{2} L_{2} \cos \left(\theta-\frac{\alpha}{2}\right)\right] \\
& +\frac{\dot{\alpha} \dot{y}}{4}\left[m_{1} L_{1} \sin \left(\theta+\frac{\alpha}{2}\right)-m_{2} L_{2} \sin \left(\theta-\frac{\alpha}{2}\right)\right] \\
& +\frac{\dot{\alpha} \dot{x}}{4}\left[m_{1} L_{1} \cos \left(\theta+\frac{\alpha}{2}\right)-m_{2} L_{2} \cos \left(\theta-\frac{\alpha}{2}\right)\right] \\
& +\frac{\ddot{\theta}}{3}\left[m_{1} L_{1}^{2}+m_{2} L_{2}^{2}\right]+\frac{\ddot{\alpha}}{6}\left[m_{1} L_{1}^{2}-m_{2} L_{2}^{2}\right] \\
& +\frac{\ddot{y}}{2}\left[m_{1} L_{1} \cos \left(\theta+\frac{\alpha}{2}\right)+m_{2} L_{2} \cos \left(\theta-\frac{\alpha}{2}\right)\right] \\
& -\frac{\ddot{x}}{2}\left[m_{1} L_{1} \sin \left(\theta+\frac{\alpha}{2}\right)+m_{2} L_{2} \sin \left(\theta-\frac{\alpha}{2}\right)\right] \\
& -\frac{\dot{y}}{2}\left[m_{1} L_{1} \sin \left(\theta+\frac{\alpha}{2}\right)\left(\dot{\theta}+\frac{\dot{\alpha}}{2}\right)\right. \\
& \left.+m_{2} L_{2} \sin \left(\theta-\frac{\alpha}{2}\right)\left(\dot{\theta}-\frac{\dot{\alpha}}{2}\right)\right] \\
& -\frac{\dot{x}}{2}\left[m_{1} L_{1} \cos \left(\theta+\frac{\alpha}{2}\right)\left(\dot{\theta}+\frac{\dot{\alpha}}{2}\right)\right. \\
+ & \left.m_{2} L_{2} \cos \left(\theta-\frac{\alpha}{2}\right)\left(\dot{\theta}-\frac{\dot{\alpha}}{2}\right)\right]
\end{aligned}
$$

4) Equation of Motion corresponding to $\alpha$ :

$$
\begin{aligned}
\frac{\tau_{1}-\tau_{2}}{2}+ & \tau=+\frac{\dot{\theta} \dot{y}}{4}\left[m_{1} L_{1} \sin \left(\theta+\frac{\alpha}{2}\right)-m_{2} L_{2} \sin \left(\theta-\frac{\alpha}{2}\right)\right] \\
& +\frac{\dot{\theta} \dot{x}}{4}\left[m_{1} L_{1} \cos \left(\theta+\frac{\alpha}{2}\right)-m_{2} L_{2} \cos \left(\theta-\frac{\alpha}{2}\right)\right] \\
& +\frac{\dot{\alpha} \dot{y}}{8}\left[m_{1} L_{1} \sin \left(\theta+\frac{\alpha}{2}\right)+m_{2} L_{2} \sin \left(\theta-\frac{\alpha}{2}\right)\right] \\
& +\frac{\dot{\alpha} \dot{x}}{8}\left[m_{1} L_{1} \cos \left(\theta+\frac{\alpha}{2}\right)+m_{2} L_{2} \cos \left(\theta-\frac{\alpha}{2}\right)\right] \\
& +\frac{\ddot{\alpha}}{12}\left[m_{1} L_{1}^{2}+m_{2} L_{2}^{2}\right]+\frac{\ddot{\theta}}{6}\left[m_{1} L_{1}^{2}-m_{2} L_{2}^{2}\right] \\
& +\frac{\ddot{y}}{4}\left[m_{1} L_{1} \cos \left(\theta+\frac{\alpha}{2}\right)-m_{2} L_{2} \cos \left(\theta-\frac{\alpha}{2}\right)\right] \\
& -\frac{\ddot{x}}{4}\left[m_{1} L_{1} \sin \left(\theta+\frac{\alpha}{2}\right)-m_{2} L_{2} \sin \left(\theta-\frac{\alpha}{2}\right)\right] \\
& -\frac{\dot{y}}{4}\left[m_{1} L_{1} \sin \left(\theta+\frac{\alpha}{2}\right)\left(\dot{\theta}+\frac{\dot{\alpha}}{2}\right)\right. \\
& \left.-m_{2} L_{2} \sin \left(\theta-\frac{\alpha}{2}\right)\left(\dot{\theta}-\frac{\dot{\alpha}}{2}\right)\right] \\
& -\frac{\dot{x}}{4}\left[m_{1} L_{1} \cos \left(\theta+\frac{\alpha}{2}\right)\left(\dot{\theta}+\frac{\dot{\alpha}}{2}\right)\right. \\
& \left.-m_{2} L_{2} \cos \left(\theta-\frac{\alpha}{2}\right)\left(\dot{\theta}-\frac{\dot{\alpha}}{2}\right)\right]
\end{aligned}
$$

where $\tau$ is the externally applied torque for actuating the scallop links.

\section{Results And Discussion}

\section{A. Simulations}

For the purpose of conducting numerical simulations and corroborating with the existing experimental results, the 
physical characteristics of the scallop were taken as denoted in the following table.

TABLE I: Microscallop Properties

\begin{tabular}{|c||c|}
\hline Scallop Property & Value \\
\hline \hline link length & $0.8 \mathrm{~mm}$ \\
\hline link breadth & $0.8 \mathrm{~mm}$ \\
\hline link thickness & $0.1 \mathrm{~mm}$ \\
\hline mass of single link & $61.76 * 10^{-9} \mathrm{~kg}$ \\
\hline Wave number & $1 \mathrm{~m}^{-1}$ \\
\hline
\end{tabular}

And the shear thinning fluid was taken to possess the following Carreau coefficients:

TABLE II: Non Newtonian Fluid Properties

\begin{tabular}{|c|c|}
\hline Fluid Property & Value \\
\hline \hline Zero strain rate viscosity & $0.377 P a-s$ \\
\hline Shear thinning coefficient $(n)$ & 0.3 \\
\hline Relaxation time & $0.512 s$ \\
\hline
\end{tabular}

The initial angle between the links is $\alpha(0)=0.5618$ radians and the initial angle between the scallop body axis and the fixed horizontal axis is $\theta(0)=0.523$ radians.

Upon simulating the four equations, the following displacement trends were observed. In all but the last case, the torque applied has a time period of 4 seconds.

1) Symmetric Actuation: With identical opening and closing speeds there is negligible net displacement as expected. Since the resistive force depends on the strain rate, the force acting during the opening and closing of the scallop remains the same if the opening and closing strain rates are similar, thereby leading to no net displacement as depicted in Fig 5 .7.

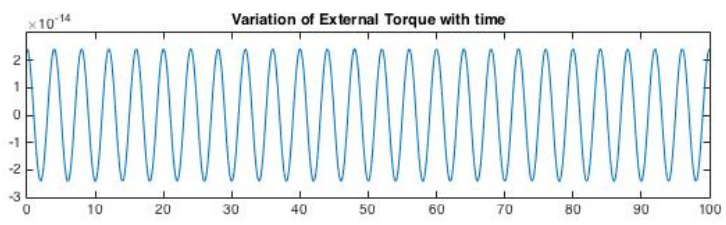

Fig. 4: Variation of the External Torque acting on the scallop

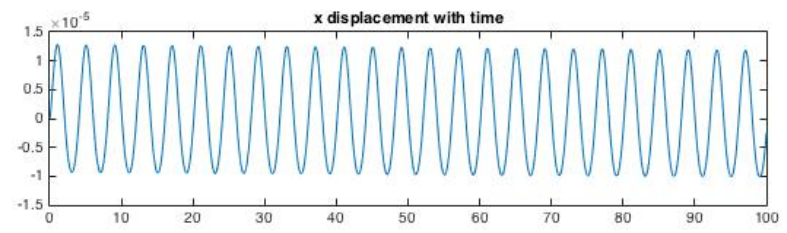

Fig. 5: Displacement in $x$ direction in $\mathrm{m} / \mathrm{s}$

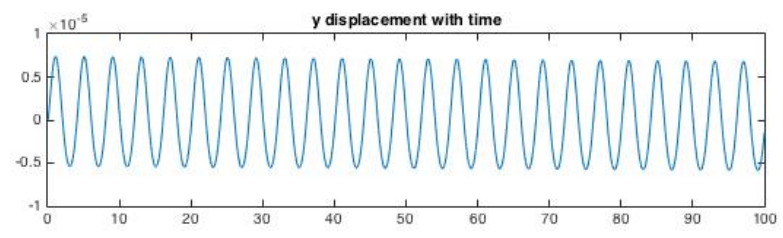

Fig. 6: Displacement in $y$ direction in $\mathrm{m} / \mathrm{s}$

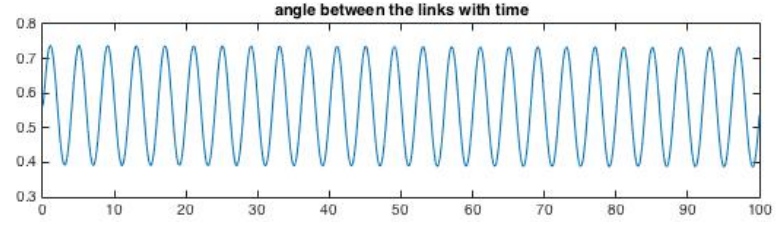

Fig. 7: Variation of $\alpha$ in rad/sec

2) Asymmetric Actuation: Upon asymmetric actuation of the scallop, with slow opening and fast closing of the links and the fluid properties as defined in Table III the displacement trends are as shown in Fig.9| 11 .

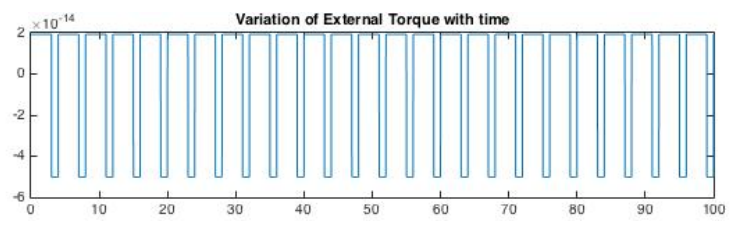

Fig. 8: Variation of the External Torque acting on the scallop

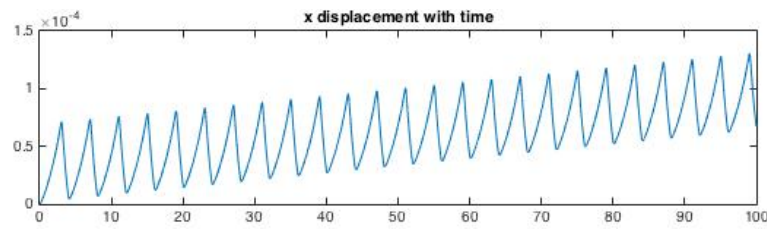

Fig. 9: Displacement in $x$ direction in $\mathrm{m} / \mathrm{s}$

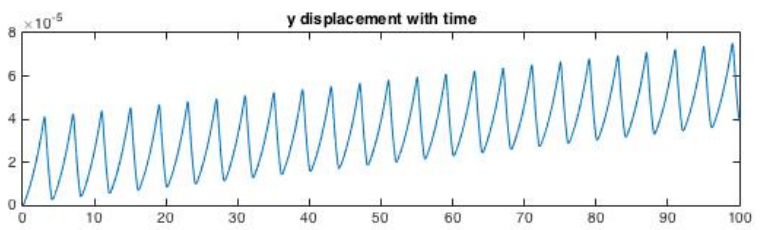

Fig. 10: Displacement in $y$ direction in $\mathrm{m} / \mathrm{s}$

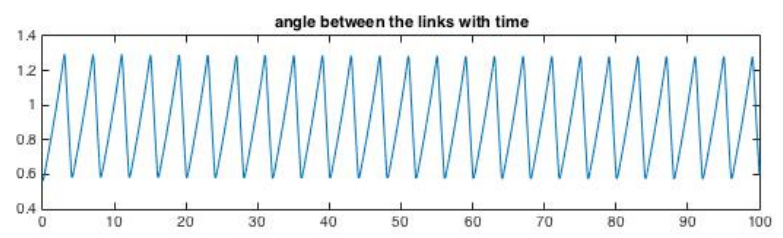

Fig. 11: Variation of $\alpha$ in $\mathrm{rad} / \mathrm{sec}$

3) Higher zero shear rate viscosity: Upon increasing the zero shear rate viscosity from $\mu_{0}=0.377$ to $\mu_{0}=$ 0.6 , under asymmetric actuation as before, the resistive force increases accordingly leading to a decrease in the overall displacement to around $60 \mu \mathrm{m}$ in $100 \mathrm{~s}$ as can be observed in Fig 12 14

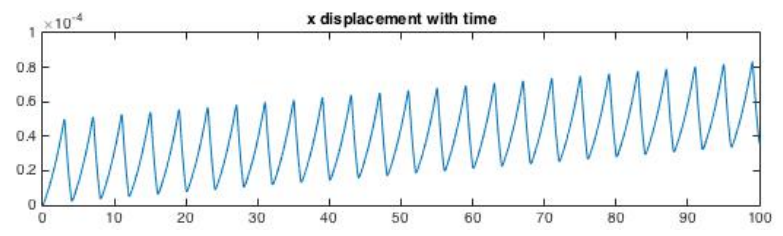

Fig. 12: Displacement in $x$ direction in $\mathrm{m} / \mathrm{s}$ 


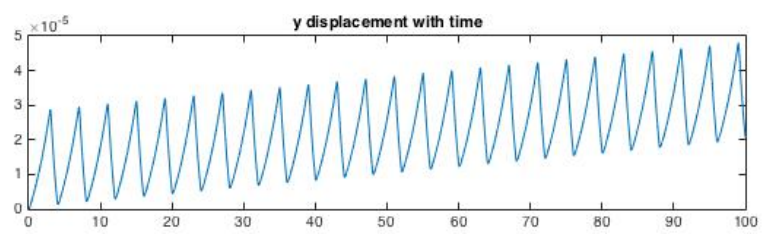

Fig. 13: Displacement in $y$ direction in $\mathrm{m} / \mathrm{s}$

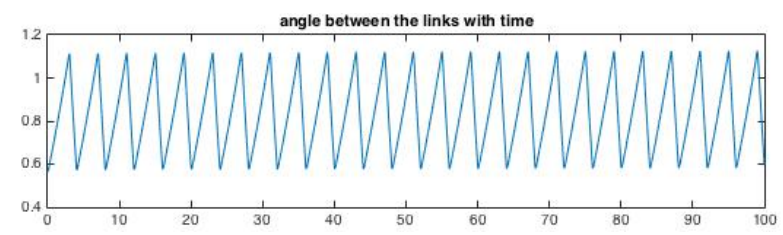

Fig. 14: Variation of $\alpha$ in $\mathrm{rad} / \mathrm{sec}$

4) Reduce shear thinning coefficient: Upon decreasing the shear thinning coefficient from $n=0.3$ to $n=0.2$, under similar asymmetric actuation, the resistive force decreases (as the shear rate has a greater effect on the viscosity with decreasing shear thinning coefficient) accordingly leading to an increase in the overall displacement to around $200 \mu \mathrm{m}$ in $100 \mathrm{~s}$ as can be observed in Fig $15,17$.

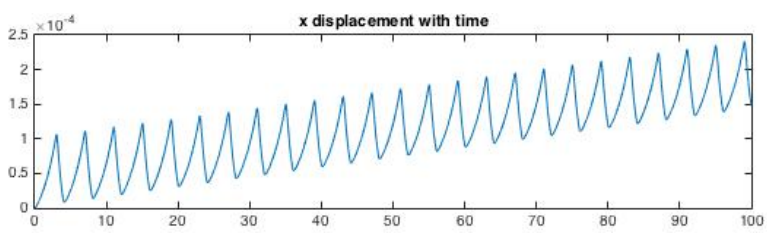

Fig. 15: Displacement in $x$ direction in $\mathrm{m} / \mathrm{s}$

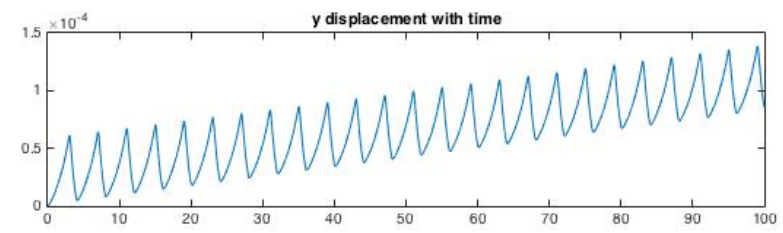

Fig. 16: Displacement in $y$ direction in $\mathrm{m} / \mathrm{s}$

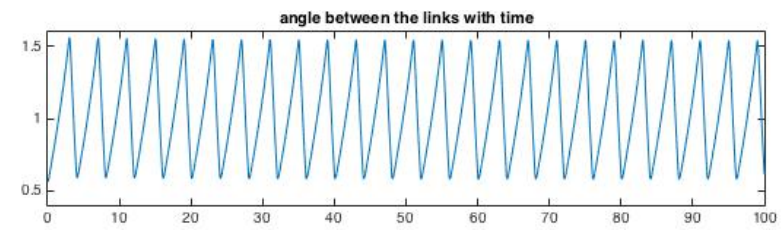

Fig. 17: Variation of $\alpha$ in $\mathrm{rad} / \mathrm{sec}$

5) Increased time period: When the time period of the actuation torque is increased from $4 s$ to $6 s$ as shown in Fig 18, the displacement obtained is greater, as the opening and closing strokes have greater velocities and thereby a lesser resistive force. This behaviour is depicted in Fig 19,21, with the fluid properties as described in Table

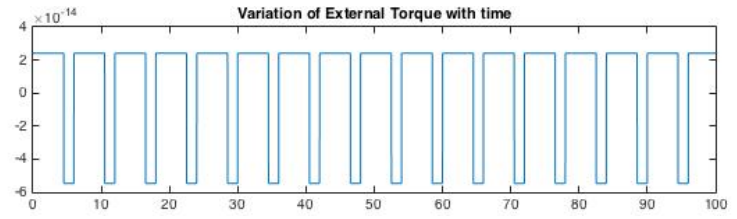

Fig. 18: Variation of the External Torque acting on the scallop

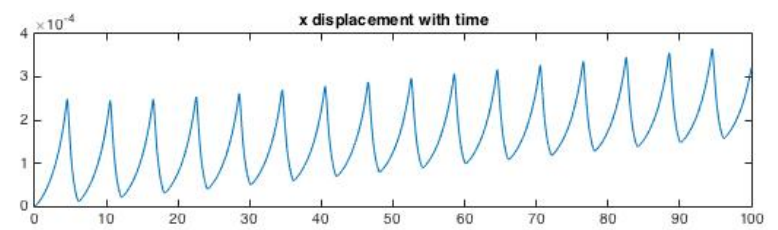

Fig. 19: Displacement in $x$ direction in $\mathrm{m} / \mathrm{s}$

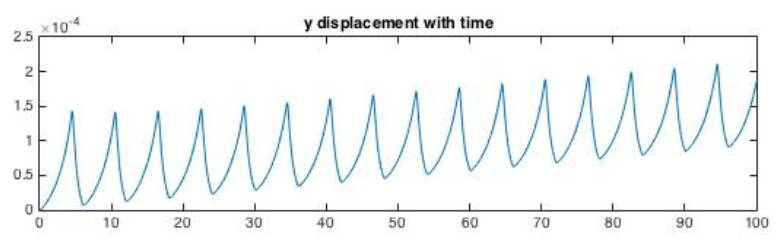

Fig. 20: Displacement in $y$ direction in $\mathrm{m} / \mathrm{s}$

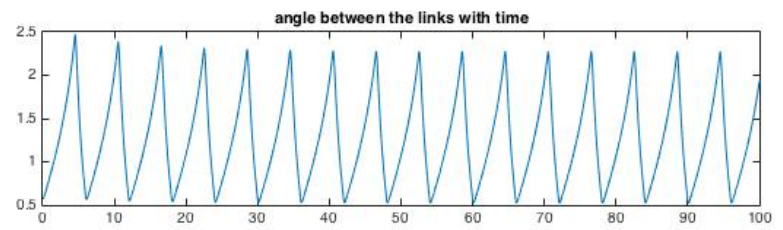

Fig. 21: Variation of $\alpha$ in $\mathrm{rad} / \mathrm{sec}$

Since the external torque actuation is of the same magnitude (the only difference being the direction each link rotates in) for both the links, the scallop traverses in a straight line with the scallop axis at a constant angle with the inertial $x$ axis as shown in the figure below.

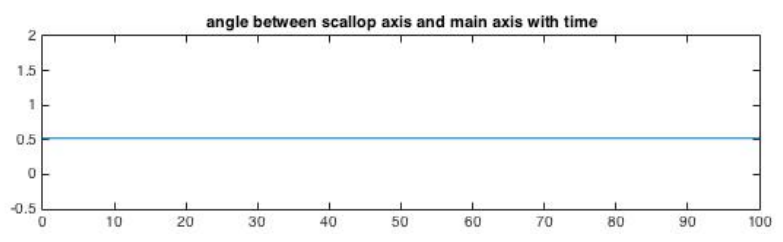

Fig. 22: Variation of $\theta$ in $\mathrm{rad} / \mathrm{sec}$

From the above simulation results, it is evident that the model adheres to the previously obtained trends in [9]. With this dynamic model at hand, we proceed to present the accessibility results of the microscallop.

\section{B. Microscallop Accessibility}

An essential objective of the modelling is to explore control algorithms for motion planning of the microscallop. In this subsection we explore a notion called accessibility at certain configurations of the microscallop. For the purpose of our simulations we have assumed the torques acting on the two links to be of equal magnitude and opposite in sign. But the two links can be subject to two different torques, 
giving rise to two control vector fields. Hence, the scallop system can be written as:

$$
\dot{x}(t)=f_{0}(x(t))+u^{1}(t) f_{1}(x(t))+u^{2}(t) f_{2}(x(t))
$$

where $x(t)$ is a curve on an eight dimensional state manifold $M$ (corresponding to the four generalised coordinates and their velocities), $u^{1}$ and $u^{2}$ are the scalar torques acting on the two links and $\left\{u^{1}, u^{2}\right\} \in U$, where $U$ is a subset in $\mathbb{R}^{2}$. The vector field $f_{0}$ is the drift vector field, describing the dynamics of the system in the absence of controls, and the vector fields $f_{1}, f_{2}$ are the input vector fields or control vector fields, indicating how we are able to actuate the system. For the above analytic control affine system, $\Sigma=\left(M, \mathscr{F}=\left\{f_{0}, f_{1}, f_{2}\right\}, U\right)$, with $U$ proper, the accessibility theorem states that $\Sigma$ is accessible from $x$ if and only if $L(\mathscr{F})_{x}=T_{x} M[14]$.
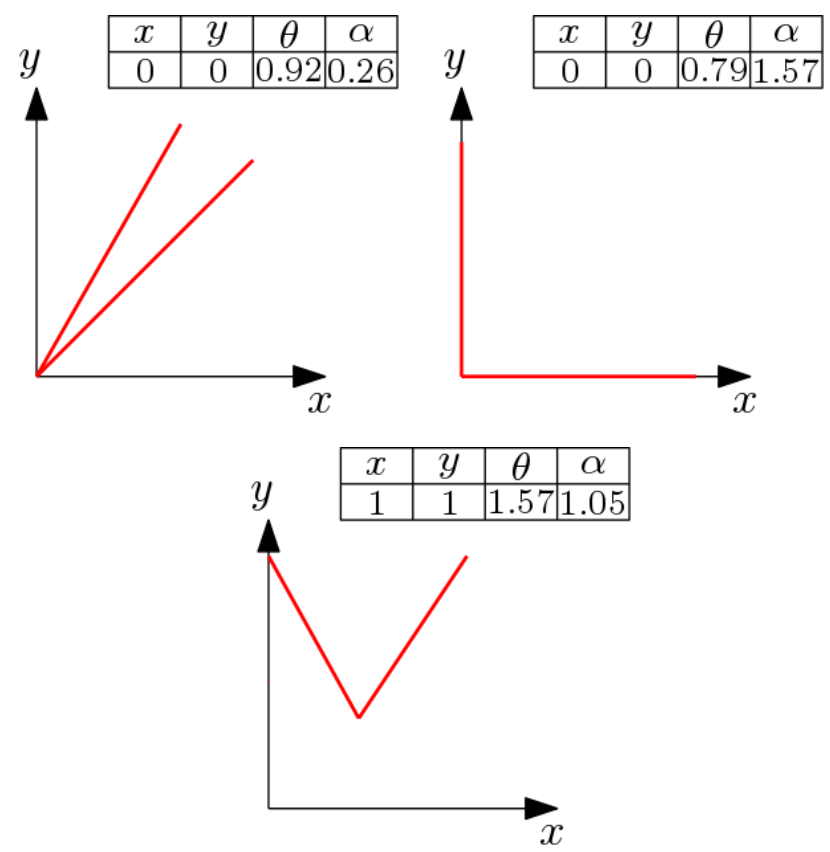

Fig. 23: Different scallop configurations at which accessibility was checked.

Accessibility was checked for the above positions of the scallop when it is in rest. The Lie brackets computed for this computation were $f_{1}, f_{2},\left[f_{0}, f_{1}\right],\left[f_{0}, f_{2}\right],\left[f_{0},\left[f_{0}, f_{1}\right]\right],\left[f_{0},\left[f_{0}, f_{2}\right]\right],\left[f_{1},\left[f_{0}, f_{1}\right]\right]$ and $\left[f_{1},\left[f_{0},\left[f_{0}, f_{1}\right]\right]\right]$, which spanned $T_{x} M$. Hence $\Sigma$ is accessible from these locations and other such configurations.

\section{CONCLUSION}

From the simulation figures we can infer that the velocity of the scallop along $\theta$ is nearly equal to $1-2 \mu \mathrm{m} / \mathrm{s}$. For a microscallop of similar dimensions and fluid properties, our technique, which is model based, predicts almost similar displacements as observed through experiments in [9]. Although we did conduct a non-dimensional analysis, it was necessary to resort to the current method in order to better understand the effect of fluid parameters on the scallop locomotion. The proposed control affine system is also accessible from various configurations. Hence, this model provides us with a means to generate other scallop motions through appropriately designed control laws.

\section{REFERENCES}

[1] Stefano Fusco, Franziska Ullrich, Juho Pokki, George Chatzipirpiridis, Berna Ozkale, Kartik M Sivaraman, Olgac Ergeneman, Salvador Pane, and Bradley J Nelson. Microrobots: a new era in ocular drug delivery. Expert Opinion on Drug Delivery, 11(11):1815-1826, 2014. PMID: 25001411.

[2] Bradley J. Nelson, Ioannis K. Kaliakatsos, and Jake J. Abbott. Microrobots for minimally invasive medicine. Annual Review of Biomedical Engineering, 12(1):55-85, jul 2010.

[3] Zhang Han, Hutmacher Dietmar W., Chollet Franck, Poo Aun Neow, and Burdet Etienne. Microrobotics and mems-based fabrication techniques for scaffold-based tissue engineering. Macromolecular Bioscience, 5(6):477-489, 2005.

[4] U. Kei Cheang and Min Jun Kim. Self-assembly of robotic micro- and nanoswimmers using magnetic nanoparticles. Journal of Nanoparticle Research, 17(3):145, Mar 2015.

[5] E. M. Purcell. Life at low reynolds number. American Journal of Physics, 45(1):3-11, 1977.

[6] L. Gary Leal and MyiLibrary. Advanced transport phenomena : Fluid Mechanics and Convective Transport processes. Cambridge ; New York : Cambridge University Press, 2007.

[7] Eric Lauga. Life around the scallop theorem. Soft Matter, 7:30603065,2011

[8] Eric Lauga and Thomas R Powers. The hydrodynamics of swimming microorganisms. Reports on Progress in Physics, 72(9):096601, 2009.

[9] Tian Qiu, Tung-Chun Lee, Andrew G. Mark, Konstantin I. Morozov, Raphael Muenster, Otto Mierka, Stefan Turek, Alexander M. Leshansky, and Peer Fischer. Swimming by reciprocal motion at low reynolds number. NATURE COMMUNICATIONS, 5, 2014. Max Planck Press Release.

[10] R.E. Johnson and C.J. Brokaw. Flagellar hydrodynamics. a comparison between resistive-force theory and slender-body theory. Biophysical Journal, 25(1):113 - 127, 1979.

[11] Emily E. Riley and Eric Lauga. Empirical resistive-force theory for slender biological filaments in shear-thinning fluids. Phys. Rev. E, 95:062416, Jun 2017.

[12] M. Anandha Rao. Flow and Functional Models for Rheological Properties of Fluid Foods, pages 27-58. Springer US, Boston, MA, 2007.

[13] Geoffrey Taylor. The action of waving cylindrical tails in propelling microscopic organisms. Proceedings of the Royal Society of London A: Mathematical, Physical and Engineering Sciences, 211(1105):225239, 1952.

[14] Andrew D. Lewis A brief on controllability of nonlinear systems, 2017 